\title{
Behavioral Interventions in Treating Anticipatory Nausea and Vomiting
}

Colmar Figueroa-Moseley, PhD; Pascal Jean-Pierre, PhD; Joseph A. Roscoe, PhD; Julie L. Ryan, PhD; Sadhna Kohli, PhD; Oxana G. Palesh, PhD; Elizabeth P. Ryan, PhD; Jennifer Carroll, MD, MPH; and Gary R. Morrow, PhD, MS, Rochester, New York

\section{Key Words}

Clinical management, anticipatory nausea and vomiting, cancer

\begin{abstract}
Anticipatory nausea and vomiting (ANV) is associated with a significant reduction in the quality of life for many chemotherapy patients. The use of 5-hydroxytryptamine type 3 receptor antagonists provides some relief for chemotherapy-induced nausea and vomiting, but does not seem to control ANV. Nonpharmacologic approaches, which include behavioral interventions, may provide the greatest promise in relieving symptoms. Little evidence supports the use of complementary and alternative methods, such as acupuncture and acupressure, in relieving ANV. Behavioral interventions, especially progressive muscle relaxation training and systematic desensitization, should be considered important methods for preventing and treating ANV. (JNCCN 2007;5:44-50)
\end{abstract}

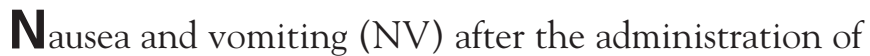
chemotherapy have been reported by patients since the drugs were first used to treat cancer. Since that time, advances in pharmacologic control of chemotherapyinduced NV have improved symptom management. However, even now, cancer patients may fear the treatment and its side effects more than the disease. Furthermore, chemotherapy-related NV is often associated with physiologic complications, such as fatigue, muscle strain, and metabolic imbalance. ${ }^{1}$ They can also contribute to inadequate caloric and fluid intake that can

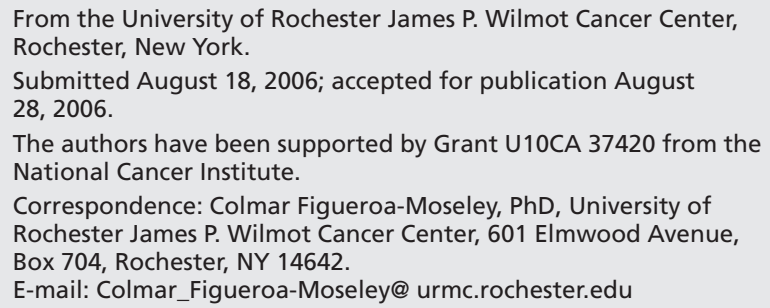

aggravate the cachexia often caused by the cancer itself. ${ }^{1}$ Finally, poorly controlled NV may lead to dose reduction or even termination of potentially curative treatment regimens..$^{2-4}$

One commonly reported consequence of the conditioning effect created by frequent or severe post-treatment $\mathrm{NV}$ is anticipatory nausea and vomiting (ANV). ANV develops in approximately $30 \%$ of patients by the fourth treatment cycle and seems to be linked to psychological, neurologic, and physiologic systems. ${ }^{5}$ Antiemetics, including the new 5-hydroxytryptamine type 3 (5-HT3) receptor antagonists, do not appear to control ANV. Furthermore, the prevalence of ANV remained unchanged after these new antiemetics were introduced. ${ }^{7}$ Fortunately, behavioral interventions have been effective in mitigating these side effects, and low doses of the anxiolytic agent, alprazolam, may be potentially useful as a pharmacologic preventive intervention. ${ }^{8}$

\section{Conditioning Mechanism of ANV}

Many studies in adult patients confirm that the development of ANV involves elements of classical conditioning. ${ }^{5,9-11}$ Classical conditioning is also known as Pavlovian conditioning, after the Russian scientist who was able to induce dogs to salivate at the sound of a bell that had been repeatedly paired with the ingestion of food. In this paradigm, an unconditioned response (salivation) that typically results from an unconditioned stimulus (the presentation of a food pellet) can be elicited by a conditioned stimulus (the ringing of a bell) that is present at the same time as the unconditioned stimulus.

This paradigm applies to chemotherapy treatment. Figure 1 shows how a conditioning model might account for the development of anticipatory side effects. 
Potential conditioned stimuli (e.g., the sight of the nurse, the room, or other sights, sounds, or smells of the clinic) are present while the unconditioned stimulus (chemotherapeutic agents) that produces the unconditioned response (nausea and emesis) are administered. Over several trials (chemotherapy cycles), the conditioned response stimuli (sights, sounds, and even thoughts of the clinic) can be learned and then produce the conditioned response of ANV. No data convincingly contradict the conclusion that ANV is conditioned. Among the supporting lines of evidence are the following:

- Anticipatory side effects do not develop unless posttreatment side effects have occurred. Few patients in our series of more than 4000 developed anticipatory nausea without experiencing posttreatment nausea at least once. The more severe the posttreatment $\mathrm{NV}$, the greater the likelihood patients developed ANV. ${ }^{12}$

- Conditioning is more successful with a greater number of repeated trials. Studies have shown that the frequency of ANV increases linearly with the number of chemotherapy cycles given. ${ }^{3,8,13,14}$ Each of the studies reports that by the fourth treatment cycle, approximately 20\% to $35 \%$ of patients experience anticipatory side effects.

Although most researchers agree that the development of ANV is strongly related to the Pavlovian conditioning model, evidence suggests that other cognitive factors, such as anxiety and response expectancies, may play an important role. Anxiety and expectancy are believed to be mediators in a patient's conditioning towards ANV. Researchers hypothesize that conditioning is one method of developing response expectancies. Kirsch et al. ${ }^{15}$ hypothesize that a change in response expectancies may, in fact, lead to a reduction of anxiety and thus reduce ANV. ${ }^{16}$ Our research unit conducted an illustrative study in $63 \mathrm{fe}$ male cancer patients with various cancer diagnoses. The patients' expectations for nausea were assessed before their first treatment on a numeric semantic rating scale, ranging from 1 ("I am certain I will not have nausea") to 5 ("I am certain I will have nausea"). Responses to this question were highly predictive of ANV before the third chemotherapy treatment $(P=$ $.001)$, with none of the women who responded with

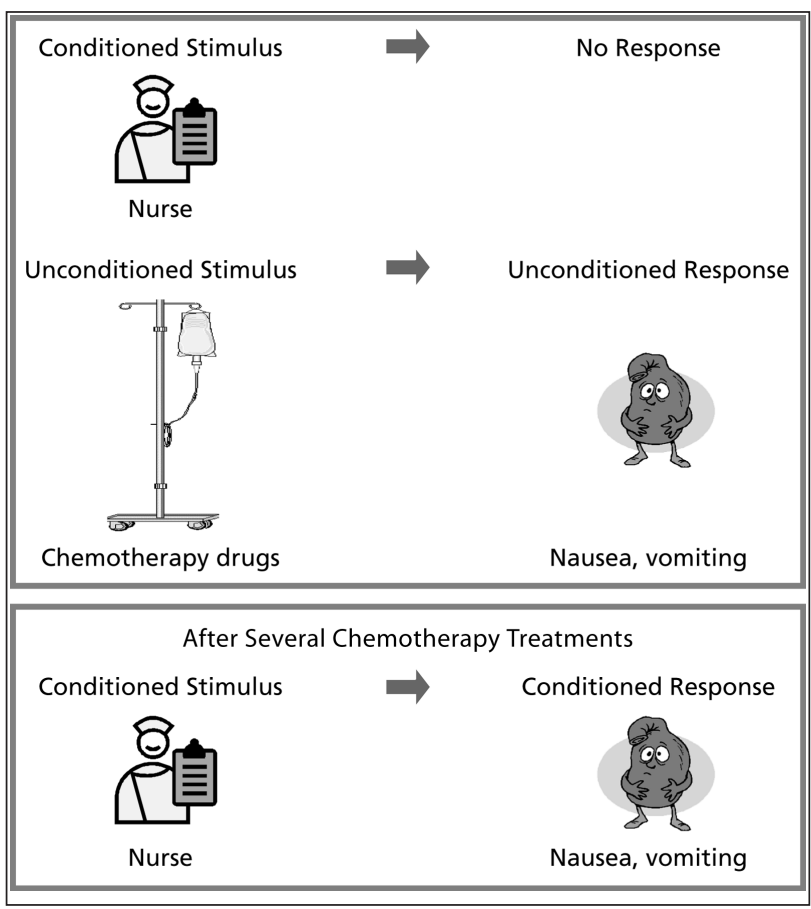

Figure 1 Components of classical conditioning.

scores of 1 or 2 developing ANV compared with 55\% of those who selected a score of $5 .{ }^{17}$

\section{Prevention and Treatment of ANV}

Traditionally, pharmacologic interventions such as 5-HT3 receptor antagonist and dexamethasone have been used to treat chemotherapy-induced NV, and have been found effective among $65 \%$ to $80 \%$ of patients. ${ }^{18}$ The need to help more patients experience emetic control has led to the use of potentially more effective pharmacologic agents, such as olanzapine, gabapentin, and cannabinoids. ${ }^{19}$

In addition, preliminary work by Razavi et al. ${ }^{8}$ suggests that low-dose alprazolam $(0.5-2 \mathrm{mg})$ ingested daily may be a potentially useful pharmacologic intervention. Their double-blind, placebo-controlled study of 57 women with breast cancer in a psychological support program, which included progressive muscle relaxation training, found that those in the placebo arm experienced a significantly higher occurrence of anticipatory nausea ( $18 \%$ vs. $0 \%)$ than those in the alprazolam arm $(P=.038)$. This observed significant difference was found in the first 2 chemotherapy cycles but not in later treatments.

In another randomized trial evaluating the efficacy of lorazepam in managing anticipatory, acute, 
Figueroa-Moseley et al.

and delayed vomiting induced by high doses of cisplatin, 180 events involving cisplatin administration were randomized to receive clemastine with and without lorazepam and metoclopramide along with dexamathasone..$^{20}$ Researchers found that lorazepam significantly reduced the incidence of ANV and acute emesis (both, $P \leq .05$ ).

Despite the potential benefits, the decision to use antiemetics is often influenced by many factors, including drug effectiveness and possible occurrence of debilitating side effects. In addition, confirmatory analyses are still needed to determine the effectiveness of antiemetics such as gabapentin and cannabinoids. ${ }^{19}$ Noticeable increases in the use of alternative and behavioral treatment of chemotherapy-induced NV have been observed recently. The noninvasiveness and lack of negative side effects of behavioral interventions have contributed to patients' willingness to use these methods to control emesis. The following sections discuss various alternative and behavioral methods that have been used to treat cancer patients.

\section{Acupuncture/Acupressure}

According to the National Institutes of Health Consensus Development Panel, acupuncture is effective in treating postoperative and chemotherapyrelated NV. A study of acupuncture and acupressure for chemotherapy-induced $\mathrm{NV}^{21}$ and an interim analysis of acupuncture against chemotherapy-induced NV in pediatric patient ${ }^{22}$ showed similar findings. No studies, however, have found any definitive evidence supporting the use of acupuncture and acupressure in alleviating ANV. Only one study provides evidence supporting the use of acupuncture in treating nervous vomiting in a dental setting. ${ }^{23}$

\section{Behavioral Treatment}

As a conditioned phenomenon, ANV is treatable through behavioral interventions based on learning principles. Research on the behavioral approaches in treating ANV has focused on 4 principal approaches: progressive muscle relaxation training (PMRT), systematic desensitization, hypnosis, and cognitive distraction.

PMRT: PMRT allows individuals to achieve a state of muscle relaxation in anticipation of or in response to various specific situations that may produce tension or anxiety, such as undergoing chemotherapy. A series of randomized clinical trials has shown the efficacy of relaxation-based behavioral treatments. ${ }^{24-26}$ The technique involves learning to relax by actively stretching and then relaxing specific muscle groups in a progressive manner. This method is often combined with guided imagery in which the individual visualizes pleasant, soothing images or scenes while relaxed. PMRT is generally taught to patients by a trained therapist, and then patients are requested to practice the technique at home using an audiotape made during the training session or a set of written instructions.

The ability of patients to put themselves in a relaxed state after one or, at most, a few therapist-directed sessions can enhance the cost-effectiveness of the procedure by limiting therapist involvement and can facilitate its continued effectiveness over time. ${ }^{27-31}$ One randomized study, however, showed that the use of an audio tape alone was not effective in teaching patients with ANV to relax before being exposed to a systematic desensitization (SD) procedure. ${ }^{32}$

PMRT seems to exert its strongest influence against adverse events, including postchemotherapy NV, anxiety, and physiologic (autonomic) arousal, that develop after chemotherapy is administered. It has been less effective against anticipatory symptoms. ${ }^{28-30,33,34}$ PMRT plus guided imagery, which also results in less nausea during chemotherapy, can prevent the development of conditioned side effects and can also decrease the frequency and severity of conditioned side effects that have already developed. ${ }^{33,35}$ It may prevent the development of ANV by decreasing anticipatory anxiety, which is believed to be a factor in the production of conditioned side effects, especially in patients using a repressive coping style. ${ }^{36}$

SD: The technique of SD, commonly used to treat learning-based difficulties such as fears and phobias, is particularly effective for treating ANV. Some phobias may develop through conditioning as previously described. In many respects, anticipatory side effects display characteristics of phobic behaviors, although the match is still far from perfect. Nonetheless, a learning-based treatment approach reduces ANV.

$\mathrm{SD}$ involves the counterconditioning of a response incompatible with the stimuli that typically elicit a maladaptive response. Applying SD to ANV, the theory predicts that anticipatory symptoms would be reduced if patients could be taught an incompatible response (e.g., muscle relaxation), rather than the conditioned response of $\mathrm{NV}$, in response to the conditioned stimuli (e.g., the clinic, the nurse). 
Treating Anticipatory Nausea and Vomiting

In this approach, patients are taught an active behavioral intervention called Jacobsonian progressive muscle relaxation. The patient actively constructs a hierarchy of stimuli that seem to elicit the ANV. This hierarchy typically involves moving progressively closer to the setting where the chemotherapy is administered. For example, patients typically start experiencing ANV a day or two before chemotherapy as they begin thinking about it. The intensity of ANV often increases on the morning of the chemotherapy visit and increases even more as patients drive to the clinic. Symptoms further increase as patients walk toward the hospital, approach the door of the treatment center, enter the room where treatment is administered, and see the drug-administration apparatus. SD operates by encouraging patients to imagine undergoing this sequence while they are deeply relaxed. Instead of learning feelings of nausea as a response to the chemotherapy experience, relaxation is counterconditioned as a response. We and other experts have found this treatment to be successful in more than half of patients in whom it has been administered. . $4,34,37-39^{-}$

Other clinical trials have shown that these behavioral techniques can be taught to oncologists and oncology nurses. We have taught this treatment approach to oncologists, nurses, and other behavioral psychologists. ${ }^{25}$ The results of this study showed that medical oncologists and nurses were as effective in using this treatment as were experienced behavioral psychologists. Therefore, with proper instruction, this treatment approach can be learned and used successfully by clinic personnel. It does, however, require time and effort that may not be available in all treatment situations.

Hypnosis: Hypnosis, also known as suggestive or trance therapy, is a self-control technique in which patients learn to invoke a physiologic state incompatible with $\mathrm{NV}$. In the method usually used to produce the altered state of consciousness, induction of total body relaxation is followed by presentation of restful psychic imagery. While hypnotized, patients can be given suggestions for specific treatment objectives, such as increasing food intake or undergoing chemotherapy..$^{40}$ Furthermore, while in hypnosis, patients can be led through a series of events (e.g., those associated with ANV) in a technique similar to SD.

Hypnosis involves a passive type of muscle relaxation and, possibly, also distraction. ${ }^{40-43}$ Although the initial sessions are usually therapist-directed, some patients can learn to induce the state themselves. Thus, a therapist need not be present during chemotherapy. ${ }^{40}$ Hypnosis has been used successfully to prevent anticipatory nausea related to chemotherapy $y^{44,45}$ and to reduce nausea after chemotherapy. ${ }^{46-48}$ Hypnosis is most often used with children and adolescents, possibly because children are more readily hypnotized than adults. ${ }^{49}$

In a controlled experiment, Cotanch et $\mathrm{al} .{ }^{41} \mathrm{ran}$ domly assigned 12 youths aged 10 to 18 years to undergo either a relaxation/self-hypnosis intervention or standard treatment. The intervention significantly reduced the frequency, severity, and duration of chemotherapy-related emesis and the intensity and duration of nausea. Oral intake was also significantly improved, and the patients reported feeling less concerned by the chemotherapy experience.

Zeltzer et al. ${ }^{50}$ compared hypnotherapy with supportive counseling and suggested that nonspecific therapy effects, such as demand characteristics or attention, may contribute in reducing NV after chemotherapy. In their study, 19 youths aged 6 to 17 years were randomly assigned to a hypnotherapy or supportive counseling group. Youths in both groups reported reductions in NV and rated chemotherapy as "less noxious" after intervention. However, no statistically or clinically significant differences in outcomes were found between the 2 approaches, whereas a later study by Zeltzer et al. ${ }^{47}$ with 54 pediatric cancer patients showed a significant reduction in the hypnotherapy group compared with the nonhypnotic distraction/relaxation group or the attention placebo (control) group.

Overall, it seems that studies using hypnosis for ANV control support the benefit of this intervention for children. Less evidence is available illustrating its usefulness for adults. To establish the effectiveness of hypnotherapy for cancer chemotherapy patients, studies with larger sample sizes are needed that randomly assign patients to treatment or appropriate control groups.

Hypnosis certainly has its advantages: it has no undesirable side effects, little training is needed to learn the induction techniques, no special equipment is needed, and it requires little physical effort on the patient's part. Misconceptions about the procedure, however, may lead some patients to refuse to try it. ${ }^{9}$ Cultural and religious factors may also play a role in patient acceptance of hypnosis. ${ }^{51}$ In summary, 
Figueroa-Moseley et al.

hypnosis can reduce anticipatory and postchemotherapy NV and improve quality of life. ${ }^{50}$

Cognitive Distraction: Cognitive distraction (CD) may be the common element responsible for the success of relaxation training and hypnosis in decreasing conditioned nausea in adult patients undergoing chemotherapy for cancer. ${ }^{42,52} \mathrm{CD}$ causes patients to focus their attention away from NV or the stimuli associated with these phenomena. ${ }^{52}$

Using commercially available video games as distraction, Redd et al..$^{52}$ examined this hypothesis in 2 studies of pediatric patients with documented ANV from cancer chemotherapy. In the first study, 26 children were randomly assigned to the video game intervention or a control group; in the second, 15 of the 26 children were studied using a multiple-baseline design in which the nausea was assessed with and without video game playing in the same group of children. In both studies, patients experienced significant reductions in conditioned nausea after video games. The changes in nausea could not be attributed to either antiemetic medication or physical relaxation (which did not occur).

In the first controlled study of CD in adults, $60 \mathrm{pa}$ tients, stratified by level of anxiety, were randomly assigned to undergo CD (video games), PMRT with guided imagery, or no intervention, and were followed up through 5 consecutive chemotherapy sessions during which antiemetic medication was kept relatively constant. Patients undergoing either intervention experienced significantly less nausea before chemotherapy than those in the control condition, and no significant differences in nausea or other outcome measures occurred between the 2 treatment groups. Anxiety level did not influence the effectiveness of either treatment modality. ${ }^{53}$ These investigators and others $^{43,52}$ concluded that $\mathrm{CD}$, by interfering with the development or expression of ANV, is responsible for at least some of the effects of PMRT on reducing this conditioned side effect.

$\mathrm{CD}$ may be an attractive alternative for pediatric patients, who sometimes have difficulty mastering relaxation and hypnosis. ${ }^{52}$ Furthermore, it could be a cost-effective method because it does not require administration by a therapist. ${ }^{52,53}$ Video games, in particular, are inexpensive effective distracters that can be individualized, require no training and minimal assistance, and can result in sustained distraction over time. ${ }^{42}$ However, the effects have been noted to de- crease over successive chemotherapy sessions. ${ }^{53}$ The effect also only persists as long as the CD technique is being used. ${ }^{17,53}$

\section{Summary}

It is apparent that multiple mechanisms operate at different points in time to cause NV after chemotherapy and that currently available pharmacologic agents may be unable to provide complete protection from ANV associated with chemotherapy treatment. A multidisciplinary approach has been advocated that includes the best possible pharmacologic control of postchemotherapy NV, adequate information provided to patients to modify their expectations, drugs prescribed as needed to decrease anxiety, and adjunctive behavioral treatment, ideally given prophylactically. ${ }^{40,54-57}$ Furthermore, if ANV is caused by a behavioral process (i.e., a classical conditioning), behavioral interventions may be particularly appropriate for treatment. In addition, behavioral interventions that prevent the development or decrease the severity of posttreatment NV may prevent the occurrence of ANV. Future studies are needed to help determine effective ways in which behavioral interventions could be integrated into the clinical practice guidelines.

\section{References}

1. Osoba D, Zee B, Warr D, et al. Effect of postchemotherapy nausea and vomiting on health-related quality of life. The Quality of Life and Symptom Control Committees of the National Cancer Institute of Canada Clinical Trials Group. Support Care Cancer 1997;5: 307-313.

2. $\overline{\text { Cooper } S}$, Georgiou V. The impact of cytotoxic chemotherapyperspectives from patients, specialists and nurses. Eur J Cancer 1992;28A[suppl 1]:S36-38.

3. Love RR, Leventhal H, Easterling DV, Nerenz DR. Side effects and emotional distress during cancer chemotherapy. Cancer 1989;63: 604-612.

4. Stewart DJ. Cancer therapy, vomiting, and antiemetics. Can J Physiol Pharmacol 1990;68:304-313.

5. Burish TG, Carey MP. Conditioned aversive responses in cancer chemotherapy patients: theoretical and developmental analysis. J Consult Clin Psychol 1986;54:593-600.

6. Parker LA, Kwiatkowska M, Mechoulam R. Delta-9-tetrahydrocannabinol and cannabidiol, but not ondansetron, interfere with conditioned retching reactions elicited by a lithium-paired context in Suncus murinus: an animal model of anticipatory nausea and vomiting. Physiol Behav 2006;87:66-71.

7. Morrow GR, Roscoe JA, Hynes HE, et al. Progress in reducing anticipatory nausea and vomiting: a study of community practice. Support Care Cancer 1998;6:46-50. 
Treating Anticipatory Nausea and Vomiting

8. Razavi D, Delvaux N, Farvacques C, et al. Prevention of adjustment disorders and anticipatory nausea secondary to adjuvant chemotherapy: a double-blind, placebo-controlled study assessing the usefulness of alprazolam. J Clin Oncol 1993;11:1384-1390.

9. Carey MP, Burish TG. Etiology and treatment of the psychological side effects associated with cancer chemotherapy: a critical review and discussion. Psychol Bull 1988;104:307-325.

10. Montgomery GH, Bovbjerg DH. The development of anticipatory nausea in patients receiving adjuvant chemotherapy for breast cancer. Physiol Behav 1997;61:737-741.

11. Morrow GR, Lindke J, Black PM. Predicting development of anticipatory nausea in cancer patients: prospective examination of eight clinical characteristics. J Pain Symptom Manage 1991;6: 215-223.

12. Roscoe JA, Morrow GR, Hickok JT, Stern RM. Nausea and vomiting remain a significant clinical problem: trends over time in controlling chemotherapy-induced nausea and vomiting in 1413 patients treated in community clinical practices. J Pain Symptom Manage 2000;20:113-121.

13. Andrykowski MA, Redd WH, Hatfield AK. Development of anticipatory nausea: a prospective analysis. J Consult Clin Psychol 1985;53:447-454.

14. Morrow GR, Hickok JT, Rosenthal SN. Progress in reducing nausea and emesis. Comparisons of ondansetron (Zofran), granisetron (Kytril), and tropisetron (Navoban). Cancer 1995;76:343-357.

15. Kirsch I, Lynn SJ, Vigorito M, Miller RR. The role of cognition in classical and operant conditioning. J Clin Psychol 2004;60:369-392.

16. Hickok JT, Roscoe JA, Morrow GR. The role of patients' expectations in the development of anticipatory nausea related to chemotherapy for cancer. J Pain Symptom Manage 2001;22:843-850.

17. Roscoe JA, Morrow GR, Hickok JT, et al. Biobehavioral factors in chemotherapy induced nausea and vomiting. J Natl Compr Canc Netw 2004;2:501-508.

18. Walton SM. Advances in use of the 5-HT3 receptor antagonists. Expert Opin Pharmacother 2000;1:207-223.

19. Sharma R, Tobin P, Clarke SJ, et al. Management of chemotherapyinduced nausea, vomiting, oral mucositis, and diarrhoea. Lancet Oncol 2005;6:93-102.

20. Malik IA, Khan WA, Qazilbash M, et al. Clinical efficacy of lorazepam in prophylaxis of anticipatory, acute, and delayed nausea and vomiting induced by high doses of cisplatin. A prospective randomized trial. Am J Clin Oncol 1995;18:170-175.

21. Collins KB, Thomas DJ. Acupuncture and acupressure for the management of chemotherapy-induced nausea and vomiting. J Am Acad Nurse Pract 2004;16:76-80.

22. Reindl TK, Geilen W, Hartmann R, et al. Acupuncture against chemotherapy-induced nausea and vomiting in pediatric oncology. Interim results of a multicenter crossover study. Support Care Cancer 2006;14:172-176.

23. Yugin $L$. Treating patients with nervous vomiting in the dental office by point-stimulating therapy. Spec Care Dentist 1989;9:27-28.

24. Morrow GR, Morrell C. Behavioral treatment for the anticipatory nausea and vomiting induced by cancer chemotherapy. $\mathrm{N}$ Engl J Med 1982;307:1476-1480.

25. Morrow GR, Asbury R, Hammon S, et al. Comparing the effectiveness of behavioral treatment for chemotherapy-induced nausea and vomiting when administered by oncologists, oncology nurses, and clinical psychologists. Health Psychol 1992;11:250-256.

26. Yoo HJ, Ahn SH, Kim SB, et al. Efficacy of progressive muscle relaxation training and guided imagery in reducing chemotherapy side effects in patients with breast cancer and in improving their quality of life. Support Care Cancer 2005;13:826-833.

27. Burish TG, Lyles JN. Effectiveness of relaxation training in reducing the aversiveness of chemotherapy in the treatment of cancer. J Behav Ther Exp Psychiatry 1979;10:357-361.

28. Burish TG, Carey MP, Krozely MG, Greco FA. Conditioned side effects induced by cancer chemotherapy: prevention through behavioral treatment. J Consult Clin Psychol 1987;55:42-48.

29. Lyles JN, Burish TG, Krozely MG, Oldham RK. Efficacy of relaxation training and guided imagery in reducing the aversiveness of cancer chemotherapy. J Consult Clin Psychol 1982;50:509-524.

30. Morrow GR, Dobkin PL. Anticipatory nausea and vomiting in cancer patients undergoing chemotherapy treatment: prevalence, etiology, and behavioral interventions. Clin Psychol Rev 1988;8:517-556.

31. Spiegel D, Spiegel H. Hypnosis in psychosomatic medicine. Psychosomatics 1980;21:35-41.

32. Morrow GR. The assessment of nausea and vomiting: past problems, current issues, and suggestions for future research. Cancer 1984;53: 2267-2278.

33. Burish TG, Jenkins RA. Effectiveness of biofeedback and relaxation training in reducing the side effects of cancer chemotherapy. Health Psychol 1992;11:17-23.

34. Morrow GR. Effect of the cognitive hierarchy in the systematic desensitization treatment of anticipatory nausea in cancer patients: a component comparison with relaxation only, counseling, and no treatment. Cognitv Ther Res 1986;10:421-446.

35. Burish TG, Vasterling JJ, Carey MP, et al. Posttreatment use of relaxation training by cancer patients. Hosp J 1988;4:1-8.

36. Lerman C, Rimer B, Blumberg B, et al. Effects of coping style and relaxation on cancer chemotherapy side effects and emotional responses. Cancer Nurs 1990;13:308-315.

37. Elam CL, Andrykowski MA. Admission interview ratings: relationship to applicant academic and demographic variables and interviewer characteristics. Acad Med 1991;66[suppl 9]:S13-15.

38. Hailey BJ, White JG. Systematic desensitization for anticipatory nausea associated with chemotherapy. Psychosomatics 1983;24: 287-291.

39. Hoffman ML. Hypnotic desensitization for the management of anticipatory emesis in chemotherapy. Am J Clin Hypn 1982;25: 173-176.

40. LaBaw W, Holton C, Tewell K, Eccles D. The use of self-hypnosis by children with cancer. Am J Clin Hypn 1975;17:233-238.

41. Cotanch $P$, Hockenberry $M$, Herman S. Self-hypnosis as antiemetic therapy in children receiving chemotherapy. Oncol Nurs Forum 1985;12:41-46.

42. Kolko DJ, Rickard-Figueroa JL. Effects of video games on the adverse corollaries of chemotherapy in pediatric oncology patients: a single-case analysis. J Consult Clin Psychol 1985;53:223-228.

43. Redd WH, Andrykowski MA. Behavioral intervention in cancer treatment: controlling aversion reactions to chemotherapy. J Consult Clin Psychol 1982;50:1018-1029.

44. Marchioro G, Azzarello G, Viviani F, et al. Hypnosis in the treatment of anticipatory nausea and vomiting in patients receiving cancer chemotherapy. Oncology 2000;59:100-104.

45. Redd WH, Andresen GV, Minagawa RY. Hypnotic control of anticipatory emesis in patients receiving cancer chemotherapy. J Consult Clin Psychol 1982;50:14-19.

46. Jacknow DS, Tschann JM, Link MP, Boyce WT. Hypnosis in the prevention of chemotherapy-related nausea and vomiting in children: a prospective study. J Dev Behav Pediatr 1994;15:258-264. 
Figueroa-Moseley et al.

47. Zeltzer LK, Dolgin MJ, LeBaron S, LeBaron C. A randomized, controlled study of behavioral intervention for chemotherapy distress in children with cancer. Pediatrics 1991;88:34-42.

48. Walker LG, Dawson AA, Pollet SM, et al. Hypnotherapy for chemotherapy side effects. British Journal of Clinical and Experimental Hypnosis 1988;5:79-82.

49. Olness K. Imagery (self-hypnosis) as adjunct therapy in childhood cancer: clinical experience with 25 patients. Am J Pediatr Hematol Oncol 1981;3:313-321.

50. Zeltzer L, LeBaron S, Zeltzer PM. The effectiveness of behavioral intervention for reduction of nausea and vomiting in children and adolescents receiving chemotherapy. J Clin Oncol 1984;2: 683-690.

51. Zeltzer L, Kellerman J, Ellenberg L, Dash J. Hypnosis for reduction of vomiting associated with chemotherapy and disease in adolescents with cancer. J Adolesc Health Care 1983;4:77-84.

52. Redd WH, Jacobsen PB, Die-Trill M, et al. Cognitive/attentional distraction in the control of conditioned nausea in pediatric cancer patients receiving chemotherapy. J Consult Clin Psychol 1987; 55:391-395.

53. Vasterling J, Jenkins RA, Tope DM, Burish TG. Cognitive distraction and relaxation training for the control of side effects due to cancer chemotherapy. J Behav Med 1993;16:65-80.

54. Andrews PLR, Sanger GJ, eds. Emesis in Anti-Cancer Therapy Mechanisms and Treatment. London: Chapman and Hall Medical, 1993.

55. Fallowfield LJ. Behavioural interventions and psychological aspects of care during chemotherapy. Eur J Cancer 1992;28A [suppl 1]:S39-41.

56. Morrow GR. Clinical characteristics associated with the development of anticipatory nausea and vomiting in cancer patients undergoing chemotherapy treatment. J Clin Oncol 1984;2: 1170-1176.

57. Morrow GR. Chemotherapy-related nausea and vomiting: etiology and management. CA Cancer J Clin 1989;39:89-104. 